\title{
Effect of keratinase on ileal amino acid digestibility in five feedstuffs fed to growing pigs
}

\author{
Chengfei Huang ${ }^{1, a}$, Dongli Ma ${ }^{1, a}$, Jianjun Zang ${ }^{1}$, Bo Zhang ${ }^{2}$, Brian Sun ${ }^{2}$, Ling Liu ${ }^{1}$, and Shuai Zhang ${ }^{1, *}$
}

* Corresponding Author: Shuai Zhang Tel: +86-10-62731109, Fax: +86-10-62733688,

E-mail: zhangshuai16@cau.edu.cn

'State Key Laboratory of Animal Nutrition, Ministry of Agriculture Feed Industry Centre, China Agricultural University, Beijing 100193, China

${ }^{2}$ Novus International, Inc, Shang Hai 200131, China

a These authors contributed equally to this work.

ORCID

Chengfei Huang

https://orcid.org/0000-0002-4943-1472

Dongli Ma

https://orcid.org/0000-0003-0800-5490

Shuai Zhang

https://orcid.org/0000-0001-5435-2640

Submitted Nov 7, 2017; Revised Feb 19, 2018; Accepted May 7, 2018
Objective: This study was conducted to evaluate the effect of keratinase (KE) on the apparent ileal digestibility (AID) and standardized ileal digestibility (SID) of amino acids (AA) in rice bran, cottonseed meal (CSM), rapeseed meal (RSM), corn distillers dried grains with solubles (DDGS), and peanut meal (PNM).

Methods: Twelve crossbred barrows (Duroc $\times$ Landrace $\times$ Yorkshire, $50.5 \pm 1.4 \mathrm{~kg}$ body weight [BW]) fitted with T-cannulas at the terminal ileum were allotted to a $12 \times 6$ Youden Square design with 12 diets and 6 periods. The treatment diets included rice bran, CSM, RSM, corn DDGS, PNM, or corn-soybean meal (cSBM) supplemented with $0.05 \% \mathrm{KE}$ or not. Diets were given to pigs at a level of $3 \% \mathrm{BW}$ in two equal meals. The endogenous AA losses were the mean results of three previously experiments determined by a same nitrogen-free diet fed to pigs. Pigs had free access to water during the experiment.

Results: The KE supplementation improved ( $\mathrm{p}<0.05$ ) the AID and SID of Met, Thr, Val, Asp, Cys, and Tyr in rice bran. Inclusion of KE increased $(p<0.05)$ the AID and SID of Met and Val in CSM. The KE supplementation decreased $(p<0.05)$ the AID and SID of His in RSM and all measured AA except for Arg, Met, Trp, Val, Gly, and Pro in corn DDGS. There was an increase $(\mathrm{p}<0.05)$ in AID and SID of Leu, Ile, Met, Ala, Cys, Ser, and Tyr in PNM supplemented with KE compared with that without KE. Inclusion of KE increased $(\mathrm{p}<0.05)$ the AID and SID of crude protein, Leu, Ile, Phe, Thr, Asp, and Ser in cSBM.

Conclusion: This study indicated that KE had different effects on ileal AA digestibility of feedstuffs for growing pigs, which can give some usage directions of KE in swine feed containing those detected feedstuffs.

Keywords: Amino Acid; Apparent Ileal Digestibility; Feedstuff; Growing Pigs; Keratinase; Standardized Ileal Digestibility

\section{INTRODUCTION}

Keratinase (KE), one of the proteases, was first isolated from the culture medium of Bacillus licheniformis PWD-1 [1,2]. Compared with most known proteases, KE displays a higher proteolytic activity [3] and can break down a wide range of proteins such as casein, collagen, elastin, and keratin as well as other proteins containing cysteine disulfide bonds [4-6]. Previous studies reported that dietary KE supplementation improved the nutrient digestibility and apparent ileal digestibility (AID) of amino acids (AA) for growing pigs [7]. It was also reported that KE could improve growth performance, breast meat yield, and gut villus structure of broilers fed diets based on corn and soybean meal (SBM) [8-10].

Soybean meal is the primary protein source of swine feed in many countries. Some soybean proteins, such as glycinin, $\beta$-conglycinin, trypsin inhibitors, lectins and other minor proteins, can negatively impact on intestinal morphology of pigs [11-13]. Cottonseed meal $(\mathrm{CSM})$, rapeseed meal (RSM), peanut meal (PNM), rice bran, and corn distillers dried grains 
with solubles (DDGS) are common local by-products that can be used in pig feed in China. However, these feedstuffs are poorly digested by pig and the nutrient values are quite varied. Improving nutritional value of these feedstuffs for pigs, such as improving AA digestibility, becomes important when they are included as lower cost alternatives in pig diets. It is reasonable to hypothesize that KE can improve the AA digestibility of those feedstuffs, which may be rich in proteins containing cysteine disulfide bonds, according to the characteristics of KE. Therefore, the main objective of this study was to investigate the effects of $\mathrm{KE}$ on the ileal digestibility of crude protein (CP) and AA in five feedstuffs of rice bran, CSM, RSM, corn DDGS, and PNM for growing pigs. In addition, the effect of KE on the corn-soybean meal (CSBM) was also evaluated because the cSBM diet is commonly used in pig feed.

\section{MATERIALS AND METHODS}

The trial protocol including animal care and use was approved by the Institutional Animal Care and Use Committee of China Agricultural University (Beijing, China).

\section{Preparation of keratinase}

The KE was provided in the premix Cibenza DP100 (Novus International, Inc., Shang Hai, China, produced in 2015) and which was produced by Bacillus licheniformis $P W D-1$, after $48 \mathrm{~h}$ fermentation at $50^{\circ} \mathrm{C}$, followed by concentrating and spray-drying [14]. The KE activity contained in this premix was more than $600,000 \mathrm{U} / \mathrm{g}$ where a unit is defined as an increase of 0.1 in absorbance at a wavelength of $280 \mathrm{~nm}$ under the conditions described by Gradišar et al [15].

\section{Animals and experimental design}

This study was conducted to evaluate the AID and standardized ileal digestibility (SID) of CP and AA in five feed feedstuffs of rice bran, CSM, RSM, corn DDGS, and PNM in the Metabolism Laboratory of the Ministry of Agriculture Feed Industry Centre (China Agricultural University, Beijing, China). A cSBM diet that is commonly used in pig feed was also evaluated in the experiment. Twelve crossbred barrows (Duroc $\times$ Landrace $\times$ Yorkshire, $50.5 \pm 1.4 \mathrm{~kg}$ body weight [BW]) fitted with Tcannula at the terminal ileum, using a method adapted from Stein et al [16], were allotted to a $12 \times 6$ Youden Square design with 6 periods and 12 diets. The experimental diets included rice bran, CSM, RSM, corn DDGS, PNM, or cSBM as the sole protein and AA source and which were each supplemented with $0.05 \%$ KE or not (Table 1). Chromic oxide was included at $0.3 \%$ as an indigestible index for calculating AA digestibility. The adjustment for basal endogenous $\mathrm{CP}$ and AA losses ( $\mathrm{g} / \mathrm{kg}$ dry matter intake) was based on estimates obtained from 3 previous studies conducted in our lab using pigs of similar BW and genetic background. The nitrogen-free diet in all 3 studies was a cornstarch-sugar-cellulose-soybean oil-based diet (73\%, $15 \%, 4 \%$, and $3 \%$ dietary inclusion, respectively). Values for endogenous $\mathrm{CP}$ and $\mathrm{AA}$ losses between the previous experiments were not different (i.e. $\mathrm{p}>0.11$ ) thus a mean value was deemed suitable. The DM, CP, and AA composition of the experimental diets is presented in Table 2. The values of endogenous CP and AA losses are presented in Table 3. Pigs were weighed at the start of each experimental period and

Table 1. Ingredient composition of the experimental diets (as-fed basis, \%)

\begin{tabular}{|c|c|c|c|c|c|c|c|c|c|c|c|c|}
\hline Ingredients & $\begin{array}{c}\text { Rice } \\
\text { bran diet }\end{array}$ & $\begin{array}{c}\text { Rice bran } \\
\text { diet+DP100 }\end{array}$ & $\begin{array}{c}\text { Cottonseed } \\
\text { meal diet }\end{array}$ & $\begin{array}{l}\text { Cottonseed } \\
\text { meal diet+ } \\
\text { DP100 }\end{array}$ & $\begin{array}{l}\text { Rapeseed } \\
\text { meal diet }\end{array}$ & $\begin{array}{c}\text { Rapeseed } \\
\text { meal diet + } \\
\text { DP100 }\end{array}$ & $\begin{array}{c}\text { Corn } \\
\text { DDGS diet }\end{array}$ & $\begin{array}{c}\text { Corn DDGS } \\
\text { diet+DP100 }\end{array}$ & $\begin{array}{l}\text { Peanut } \\
\text { meal diet }\end{array}$ & $\begin{array}{l}\text { Peanut } \\
\text { meal diet } \\
\text { +DP100 }\end{array}$ & $\begin{array}{c}\text { Corn } \\
\text { SBM diet }\end{array}$ & $\begin{array}{c}\text { Corn SBM } \\
\text { diet } \\
+ \text { DP100 }\end{array}$ \\
\hline Rice bran & 69.97 & 69.95 & - & - & - & - & - & - & - & - & - & - \\
\hline Cottonseed meal & - & - & 37.98 & 37.98 & - & - & - & - & - & - & - & - \\
\hline Canola meal & - & - & - & - & 43.48 & 43.48 & - & - & - & - & - & - \\
\hline Corn DDGS & - & - & - & - & - & - & 58.97 & 58.97 & - & - & - & - \\
\hline Peanut meal & - & - & - & - & - & - & - & - & 34.98 & 34.98 & - & - \\
\hline Corn starch & 15.73 & 15.75 & 37.72 & 37.72 & 32.22 & 32.22 & 26.73 & 26.73 & 45.72 & 45.72 & - & - \\
\hline Corn & - & - & - & - & - & - & - & - & - & - & 69.97 & 69.97 \\
\hline Soybean meal & - & - & - & - & - & - & - & - & - & - & 25.73 & 25.73 \\
\hline Sucrose & 10.00 & 10.00 & 20.00 & 20.00 & 20.00 & 20.00 & 10.00 & 10.00 & 15.00 & 15.00 & - & - \\
\hline Dicalcium phosphate & 3.00 & 3.00 & 3.00 & 3.00 & 3.00 & 3.00 & 3.00 & 3.00 & 3.00 & 3.00 & 3.00 & 3.00 \\
\hline Sodium chloride & 0.45 & 0.45 & 0.45 & 0.45 & 0.45 & 0.45 & 0.45 & 0.45 & 0.45 & 0.45 & 0.45 & 0.45 \\
\hline Chromic oxide & 0.30 & 0.30 & 0.30 & 0.30 & 0.30 & 0.30 & 0.30 & 0.30 & 0.30 & 0.30 & 0.30 & 0.30 \\
\hline DP100 & - & 0.05 & - & 0.05 & - & 0.05 & - & 0.05 & - & 0.05 & - & 0.05 \\
\hline Starch & 0.05 & 0.00 & 0.05 & 0.00 & 0.05 & 0.00 & 0.05 & 0.00 & 0.05 & 0.00 & 0.05 & 0.00 \\
\hline Vit-min premix & 0.50 & 0.50 & 0.50 & 0.50 & 0.50 & 0.50 & 0.50 & 0.50 & 0.50 & 0.50 & 0.50 & 0.50 \\
\hline Total & 100.00 & 100.00 & 100.00 & 100.00 & 100.00 & 100.00 & 100.00 & 100.00 & 100.00 & 100.00 & 100.00 & 100.00 \\
\hline
\end{tabular}

DDGS, distillers dried grains with solubles; SBM, soybean meal.

1) DP100: Keratinase, provided by Novus International, Inc.

2) Vit-min premix provided the following quantities of vitamins and microminerals per kilogram of the diet for pigs: vitamin $A, 5,600$ IU; vitamin $D_{3}, 2,200$ IU; vitamin $E, 21.6$

IU; vitamin $\mathrm{K}_{3}, 1.8 \mathrm{mg}$; vitamin $\mathrm{B}_{12}, 12 \mu \mathrm{g}$; thiamine, $0.88 \mathrm{mg}$; riboflavin, 4 mg; pantothenic acid, $10 \mathrm{mg}$; niacin, 20 mg; choline chloride, $0.32 \mathrm{~g}$; folacin, 0.4 mg; pyridoxine, 1.8 mg; biotin, $40 \mu g ; \mathrm{Fe}\left(\mathrm{FeSO}_{4} \cdot \mathrm{H}_{2} \mathrm{O}\right), 88 \mathrm{mg} ; \mathrm{Cu}\left(\mathrm{CuSO}_{4} \cdot 5 \mathrm{H}_{2} \mathrm{O}\right), 120 \mathrm{mg} ; \mathrm{Zn}(\mathrm{ZnO}), 96 \mathrm{mg} ; \mathrm{Mn}(\mathrm{MnO}), 16 \mathrm{mg} ; \mathrm{I}(\mathrm{KI}), 0.24 \mathrm{mg} ;$ and Se $\left(\mathrm{Na}_{2} \mathrm{SeO}\right)_{3}, 0.4 \mathrm{mg}$. 
Table 2. Analyzed composition of the experimental diets (as-fed basis, \%)

\begin{tabular}{|c|c|c|c|c|c|c|c|c|c|c|c|c|}
\hline Item & $\begin{array}{c}\text { Rice } \\
\text { bran diet }\end{array}$ & $\begin{array}{c}\text { Rice bran } \\
\text { diet+DP100 }\end{array}$ & $\begin{array}{c}\text { Cottonseed } \\
\text { meal diet }\end{array}$ & $\begin{array}{l}\text { Cottonseed } \\
\text { meal diet } \\
\text { +DP100 }\end{array}$ & $\begin{array}{l}\text { Rapeseed } \\
\text { meal diet }\end{array}$ & $\begin{array}{l}\text { Rapeseed } \\
\text { meal diet }\end{array}$ & $\begin{array}{c}\text { Corn } \\
\text { DDGS diet }\end{array}$ & $\begin{array}{c}\text { Corn } \\
\text { DDGS } \\
\text { diet+DP100 }\end{array}$ & $\begin{array}{c}\text { Peanut } \\
\text { meal diet }\end{array}$ & $\begin{array}{l}\text { Peanut meal } \\
\text { diet +DP100 }\end{array}$ & $\begin{array}{c}\text { Corn } \\
\text { SBM diet }\end{array}$ & $\begin{array}{c}\text { Corn } \\
\text { SBM diet } \\
\text { +DP100 }\end{array}$ \\
\hline Dry matter & 89.54 & 89.81 & 91.08 & 91.05 & 91.27 & 91.16 & 90.02 & 90.50 & 89.86 & 90.88 & 88.31 & 88.41 \\
\hline Crude protein & 11.20 & 11.17 & 19.47 & 19.91 & 18.83 & 18.59 & 17.81 & 18.18 & 20.53 & 20.00 & 20.03 & 20.57 \\
\hline \multicolumn{13}{|c|}{ Indispensable amino acid } \\
\hline $\operatorname{Arg}$ & 0.77 & 0.74 & 2.17 & 2.12 & 1.02 & 0.96 & 0.70 & 0.72 & 1.98 & 2.17 & 1.19 & 1.10 \\
\hline His & 0.32 & 0.31 & 0.53 & 0.57 & 0.56 & 0.43 & 0.52 & 0.44 & 0.48 & 0.55 & 0.56 & 0.55 \\
\hline lle & 0.84 & 0.81 & 1.21 & 1.19 & 1.50 & 1.37 & 2.50 & 2.44 & 1.39 & 1.52 & 1.98 & 2.01 \\
\hline Leu & 0.34 & 0.33 & 0.60 & 0.58 & 0.80 & 0.67 & 0.60 & 0.58 & 0.60 & 0.72 & 0.78 & 0.82 \\
\hline Lys & 0.54 & 0.52 & 0.74 & 0.69 & 0.98 & 0.93 & 0.38 & 0.32 & 0.54 & 0.58 & 0.94 & 0.98 \\
\hline Met & 0.11 & 0.15 & 0.16 & 0.18 & 0.28 & 0.23 & 0.23 & 0.26 & 0.10 & 0.12 & 0.21 & 0.18 \\
\hline Phe & 0.44 & 0.42 & 1.02 & 1.00 & 0.65 & 0.64 & 0.87 & 0.85 & 1.01 & 1.11 & 0.99 & 0.98 \\
\hline Thr & 0.42 & 0.42 & 0.57 & 0.57 & 0.78 & 0.72 & 0.61 & 0.61 & 0.53 & 0.59 & 0.73 & 0.74 \\
\hline Trp & 0.12 & 0.13 & 0.21 & 0.21 & 0.26 & 0.25 & 0.11 & 0.11 & 0.17 & 0.19 & 0.24 & 0.21 \\
\hline Val & 0.50 & 0.51 & 0.77 & 0.89 & 1.09 & 0.91 & 0.84 & 0.82 & 0.79 & 0.95 & 1.03 & 1.05 \\
\hline \multicolumn{13}{|c|}{ Dispensable amino acid } \\
\hline Ala & 0.63 & 0.62 & 0.70 & 0.72 & 0.85 & 0.80 & 1.30 & 1.29 & 0.77 & 0.84 & 1.00 & 1.02 \\
\hline Asp & 0.97 & 0.95 & 1.68 & 1.63 & 1.23 & 1.16 & 1.10 & 1.09 & 2.17 & 2.39 & 1.84 & 1.92 \\
\hline Cys & 0.17 & 0.20 & 0.25 & 0.25 & 0.45 & 0.41 & 0.30 & 0.30 & 0.21 & 0.21 & 0.29 & 0.29 \\
\hline Glu & 1.26 & 1.20 & 3.35 & 3.39 & 3.06 & 2.85 & 3.02 & 3.01 & 3.48 & 3.82 & 3.25 & 3.39 \\
\hline Gly & 0.55 & 0.53 & 0.75 & 0.71 & 0.91 & 0.84 & 0.64 & 0.65 & 1.09 & 1.18 & 0.75 & 0.78 \\
\hline Pro & 0.54 & 0.46 & 0.88 & 0.76 & 1.30 & 1.19 & 1.51 & 1.55 & 0.98 & 1.11 & 1.27 & 1.30 \\
\hline Ser & 0.48 & 0.46 & 0.71 & 0.73 & 0.75 & 0.70 & 0.76 & 0.79 & 0.85 & 0.92 & 0.88 & 0.91 \\
\hline Tyr & 0.21 & 0.21 & 0.49 & 0.36 & 0.32 & 0.27 & 0.43 & 0.41 & 0.37 & 0.60 & 0.31 & 0.32 \\
\hline
\end{tabular}

DDGS, distillers dried grains with solubles; SBM, soybean meal.

1) Data were analyzed in duplicates.

${ }^{2)}$ DP100: Keratinase, provided by Novus International, Inc.

Table 3. Basal endogenous CP and AA losses based on estimates obtained from 3 previous studies ( $\mathrm{g} / \mathrm{kg}$ dry matter intake) ${ }^{1)}$

\begin{tabular}{|c|c|c|c|c|c|c|}
\hline Items & Average & 1st study & 2nd study & 3rd study & SEM & $\mathrm{p}$-value \\
\hline $\mathrm{CP}$ & 8.73 & 8.61 & 9.06 & 8.62 & 1.42 & 0.97 \\
\hline \multicolumn{7}{|c|}{ Indispensable amino acid } \\
\hline Arg & 0.25 & 0.19 & 0.14 & 0.31 & 0.07 & 0.23 \\
\hline His & 0.08 & 0.12 & 0.08 & 0.06 & 0.01 & 0.12 \\
\hline lle & 0.22 & 0.25 & 0.22 & 0.21 & 0.04 & 0.65 \\
\hline Leu & 0.46 & 0.42 & 0.36 & 0.54 & 0.08 & 0.29 \\
\hline Lys & 0.28 & 0.29 & 0.26 & 0.29 & 0.05 & 0.90 \\
\hline Met & 0.07 & 0.11 & 0.09 & 0.10 & 0.03 & 0.81 \\
\hline Phe & 0.41 & 0.30 & 0.45 & 0.46 & 0.07 & 0.23 \\
\hline Thr & 0.35 & 0.36 & 0.33 & 0.35 & 0.06 & 0.93 \\
\hline Trp & 0.08 & 0.08 & 0.10 & 0.08 & 0.01 & 0.45 \\
\hline \multicolumn{7}{|c|}{ Dispensable amino acid } \\
\hline Ala & 0.40 & 0.41 & 0.33 & 0.44 & 0.09 & 0.65 \\
\hline Asp & 0.49 & 0.45 & 0.46 & 0.53 & 0.09 & 0.77 \\
\hline Cys & 0.25 & 0.26 & 0.26 & 0.24 & 0.04 & 0.78 \\
\hline Glu & 0.68 & 0.68 & 0.72 & 0.66 & 0.13 & 0.92 \\
\hline Gly & 0.67 & 0.58 & 0.49 & 0.78 & 0.22 & 0.53 \\
\hline Pro & 0.39 & 0.38 & 0.38 & 0.40 & 0.16 & 0.97 \\
\hline Ser & 0.29 & 0.27 & 0.29 & 0.29 & 0.05 & 0.96 \\
\hline Tyr & 0.14 & 0.12 & 0.15 & 0.10 & 0.04 & 0.86 \\
\hline Val & 0.37 & 0.29 & 0.38 & 0.40 & 0.06 & 0.50 \\
\hline
\end{tabular}

$\mathrm{CP}$, crude protein; $\mathrm{AA}$, amino acids; $\mathrm{SEM}$, standard error of means.

1) The 3 studies were conducted in the same lab using pigs of similar body weight and genetic background (unpublished data). The nitrogen-free diet used in the 3 studies have same formulations. These data were comparable with the values from previous studies that also conducted in our lab $[20,24,27]$. feed allotment adjusted accordingly. All pigs had free access to water during the experiment.

\section{Digesta collection and sampling}

Each experimental period lasted for $7 \mathrm{~d}$ where the first $5 \mathrm{~d}$ were considered the adaptation period followed by $2 \mathrm{~d}$ of digesta collection. Digesta was collected continuously from 08:00 to 17:00 using procedures described by Stein et al [16]. Digesta was collected in plastic bags attached to the simple T-cannula. Bags were changed when digesta made up no more than 30\% of the bag volume. Collected digesta was immediately stored in a $-20^{\circ} \mathrm{C}$ freezer.

At the end of the experiment, ileal digesta was thawed and mixed within animal and diet, and a sub-sample of $500 \mathrm{~mL}$ was taken. Digesta samples were lyophilized in a vacuum-freeze dryer (Tofflon Freezing Drying Systems, Minhang District, Shanghai, China) and ground through a 1-mm screen for further chemical analysis.

\section{Chemical analyses}

The DM, CP, and AA were analyzed in the feedstuffs, diets, and digesta. Feedstuffs were also analyzed for ether extract (EE), crude fiber (CF), neutral detergent fiber (NDF), acid detergent fiber (ADF) and ash. All experimental diets and digesta were analyzed for $\mathrm{Cr}$ to calculate the concentration of indigestible index chromic oxide. The DM, CP, and ash in samples were analyzed according to AOAC procedures (method 
930.15, DM; method 984.13, CP; method 942.05, ash) [17] and EE was determined according to Thiex et al [18]. The CF, NDF, and $\mathrm{ADF}$ were determined using filter bags and fiber analyzer equipment (Fiber Analyzer, Ankom Technology, Macedon, NY, USA) following the procedures described by Van Soest et al [19]. The concentration of NDF was analyzed using heat stable $\alpha$-amylase and sodium sulfite without correction for insoluble ash.

Determination of AA was conducted according to $\mathrm{Li}$ et al [20] where samples were hydrolyzed with $6 \mathrm{~N} \mathrm{HCl}$ at $110^{\circ} \mathrm{C}$ for $24 \mathrm{~h}$ and then analyzed using an Amino Acid Analyzer (Hitachi L-8900, Tokyo, Japan). Sulfur-AA (Met and Cys) content of digesta, diets, and feedstuffs was determined using cold performic acid oxidation overnight and hydrolyzed with 7.5 $\mathrm{N} \mathrm{HCl}$ at $110^{\circ} \mathrm{C}$ for $24 \mathrm{~h}$ before measurement using an Amino Acid Analyzer (Hitachi L-8900, Japan). Estimates of Trp were made by hydrolyzing samples with $\mathrm{LiOH}$ for $22 \mathrm{~h}$ at a constant temperature of $110^{\circ} \mathrm{C}$ and then analyzed using High Performance Liquid Chromatography (Agilent 1200 Series, Santa Clara, CA, USA). Analysis of the $\mathrm{Cr}$ concentration in all diets and digesta was conducted using a polarized Zeeman Atomic
Absorption Spectrometer (Hitachi Z2000, Japan) after nitric acid-perchloric acid wet ash sample preparation. All chemical analyses were conducted in two duplicates. The analyzed composition of feedstuffs is presented in Table 4 .

\section{Calculations}

The AID of AA was calculated using the following method described by Stein et al [21]:

$$
\mathrm{AID}=\left[1-\left(\mathrm{AA}_{\mathrm{d}} / \mathrm{AA}_{\mathrm{f}}\right) \times\left(\mathrm{Cr}_{\mathrm{f}} / \mathrm{Cr}_{\mathrm{d}}\right)\right] \times 100 \%
$$

Where, $\mathrm{AA}_{\mathrm{d}}$ and $\mathrm{Cr}_{\mathrm{d}}$ were the concentrations of $\mathrm{AA}$ and $\mathrm{Cr}$ in the ileal digesta $(\mathrm{g} / \mathrm{kg}$ of $\mathrm{DM})$ and $\mathrm{AA}_{\mathrm{f}}$ and $\mathrm{Cr}_{\mathrm{f}}$ were the concentrations of $\mathrm{AA}$ and $\mathrm{Cr}$ in the test diets ( $\mathrm{g} / \mathrm{kg}$ of $\mathrm{DM})$. The SID of AA was calculated using the following equation:

$$
\operatorname{SID}=\left[\mathrm{AID}+\left(\mathrm{IAA}_{\text {end }} / \mathrm{AA}_{\mathrm{f}}\right) \times 100 \%\right]
$$

In which $\mathrm{IAA}_{\text {end }}$ is the basal endogenous loss of an $\mathrm{AA}(\mathrm{g} / \mathrm{kg}$ of DM intake). The AID and SID of CP were determined using the same two aforementioned equations.

\begin{tabular}{|c|c|c|c|c|c|c|c|}
\hline Items & Rice bran & Cottonseed meal & Rapeseed meal & Corn DDGS & Peanut meal & Corn & Soybean meal \\
\hline \multicolumn{8}{|c|}{ Proximate composition } \\
\hline $\mathrm{DM}$ & 90.07 & 90.78 & 88.06 & 90.78 & 90.87 & 87.21 & 90.30 \\
\hline$C P$ & 13.42 & 50.76 & 38.75 & 27.61 & 51.54 & 8.26 & 47.46 \\
\hline $\mathrm{EE}$ & 16.60 & 0.50 & 1.16 & 8.12 & 3.15 & 3.19 & 2.40 \\
\hline $\mathrm{CF}$ & 9.63 & 14.08 & 10.53 & 8.77 & 5.12 & 2.46 & 5.71 \\
\hline NDF & 21.45 & 26.70 & 24.49 & 31.47 & 16.76 & 12.95 & 11.99 \\
\hline ADF & 9.39 & 17.70 & 17.04 & 13.50 & 8.35 & 2.84 & 6.45 \\
\hline Ash & 8.70 & 6.29 & 6.61 & 4.28 & 6.12 & 1.13 & 6.25 \\
\hline \multicolumn{8}{|c|}{ Indispensable amino acid } \\
\hline $\operatorname{Arg}$ & 0.95 & 5.24 & 2.11 & 0.91 & 5.46 & 0.30 & 3.24 \\
\hline His & 0.36 & 1.25 & 1.08 & 0.74 & 1.23 & 0.22 & 1.27 \\
\hline Ile & 0.50 & 1.30 & 1.40 & 0.86 & 1.61 & 0.27 & 2.04 \\
\hline Leu & 1.05 & 2.74 & 2.88 & 3.78 & 3.60 & 1.25 & 3.94 \\
\hline Lys & 0.70 & 1.81 & 2.20 & 0.56 & 1.53 & 0.23 & 2.93 \\
\hline Met & 0.18 & 0.41 & 0.55 & 0.42 & 0.30 & 0.12 & 0.41 \\
\hline Phe & 0.57 & 2.34 & 1.32 & 1.26 & 2.60 & 0.39 & 2.41 \\
\hline Thr & 0.51 & 1.40 & 1.59 & 0.92 & 1.36 & 0.28 & 1.84 \\
\hline $\operatorname{Trp}$ & 0.14 & 0.53 & 0.55 & 0.16 & 0.48 & 0.06 & 0.59 \\
\hline Val & 0.66 & 1.95 & 1.94 & 1.21 & 2.00 & 0.34 & 2.33 \\
\hline \multicolumn{8}{|c|}{ Dispensable amino acid } \\
\hline Ala & 0.76 & 1.64 & 1.61 & 1.85 & 1.96 & 0.56 & 1.98 \\
\hline Asp & 1.21 & 4.02 & 2.53 & 1.61 & 5.83 & 0.53 & 5.29 \\
\hline Cys & 0.26 & 0.66 & 0.88 & 0.51 & 0.57 & 0.15 & 0.59 \\
\hline Glu & 1.57 & 8.01 & 5.97 & 4.42 & 9.02 & 1.40 & 7.88 \\
\hline Gly & 0.69 & 1.73 & 1.83 & 0.94 & 2.93 & 0.29 & 1.96 \\
\hline Pro & 0.69 & 1.85 & 2.45 & 2.21 & 2.35 & 0.79 & 2.50 \\
\hline Ser & 0.55 & 1.71 & 1.46 & 1.12 & 2.15 & 0.35 & 2.21 \\
\hline Tyr & 0.34 & 0.98 & 0.78 & 0.81 & 1.56 & 0.22 & 1.29 \\
\hline
\end{tabular}

Table 4. Analyzed composition of the ingredients used in the experiment (as-fed basis, \%)

DDGS, distillers dried grains with solubles; DM, dry matter; $E E$, ether extract; $C F$, crude fiber; NDF, neutral detergent fiber; ADF, acid detergent fiber.

1) Data were analyzed in duplicates. 


\section{Statistical analysis}

Data were checked for normality, and outliers were detected and then removed using the UNIVARIATE procedure of SAS (SAS Inst. Inc., Cary, NC, USA). Outliers were defined as those beyond the range of mean \pm 3 times standard deviation. Data were then analyzed using MIXED procedure of SAS, and the statistical model included the fixed effects of feedstuff and KE supplementation, and their interaction effect, and the random effects of animal and period. Pig was treated as the experimental unit. Means were calculated using the LSMEANS statement, and multiple comparison were adjusted using Tukey's test. Because the interaction effects between feedstuff and KE were significant for almost all the parameters we tested (AID and SID of AA), data were then analyzed within each feedstuff by one-way analysis of variance using the general linear model procedure of SAS to test the effects of $\mathrm{KE}$ within each ingredient. An a value of 0.05 was used to assess statistical significances among treatment means.

\section{RESULTS}

The results of AID and SID of CP and AA are shown in Tables 5,6 , respectively. There was a significant interaction effect between feedstuff and KE supplementation for AID and SID of

Table 5. Apparent ileal digestibility (AID) of crude protein (CP) and amino acids (AA) in 6 experimental diets with or without keratinase addition fed to pigs (\%) $)^{1)}$

\begin{tabular}{|c|c|c|c|c|c|c|c|c|c|c|c|c|c|c|c|c|c|c|c|}
\hline Items & $\mathrm{CP}$ & Arg & His & Ile & Leu & Lys & Met & Phe & Thr & Trp & Val & Ala & Asp & Cys & Glu & Gly & Pro & Ser & Tyr \\
\hline \multicolumn{20}{|l|}{ Diets } \\
\hline Rice bran & $53.9^{d}$ & $82.3^{c}$ & $68.3^{c}$ & $58.5^{e}$ & $66.0^{e}$ & $63.6^{c}$ & $62.9^{d}$ & $62.1^{d}$ & $54.2^{c}$ & $63.0^{b}$ & $56.2^{d}$ & $59.6^{d}$ & $59.2^{c}$ & $47.1^{c}$ & $69.8^{d}$ & $36.9^{b}$ & $50.3^{b}$ & $56.9^{e}$ & $59.9^{d}$ \\
\hline $\begin{array}{l}\text { Cottonseed } \\
\text { meal }\end{array}$ & $73.9^{b}$ & $91.4^{\mathrm{ab}}$ & $72.2^{b c}$ & $76.9^{c}$ & $78.2^{d}$ & $62.8^{c}$ & $69.8^{c}$ & $85.3^{b}$ & $68.2^{b}$ & $76.7^{\mathrm{a}}$ & $78.6^{b}$ & $66.9^{c}$ & $80.6^{a}$ & $72.3^{\mathrm{a}}$ & $86.7^{\mathrm{ab}}$ & $63.4^{\mathrm{a}}$ & $53.1^{b}$ & $75.6^{\mathrm{bc}}$ & $78.9^{b}$ \\
\hline Rapeseed meal & $70.1^{\mathrm{b}}$ & $82.9^{c}$ & $77.6^{\mathrm{b}}$ & $78.3^{\mathrm{bc}}$ & $79.5^{\text {cd }}$ & $75.7^{b}$ & $87.0^{b}$ & $78.5^{c}$ & $69.3^{b}$ & $68.2^{b}$ & $78.6^{b}$ & $75.2^{\mathrm{ab}}$ & $71.6^{\mathrm{b}}$ & $77.5^{\mathrm{a}}$ & $84.7^{b}$ & $65.8^{\mathrm{a}}$ & $67.5^{\mathrm{ab}}$ & $71.8^{\mathrm{cd}}$ & $70.1^{c}$ \\
\hline Corn DDGS & $61.3^{c}$ & $80.4^{c}$ & $70.4^{c}$ & $69.2^{d}$ & $81.9^{c}$ & $41.5^{d}$ & $87.0^{b}$ & $79.6^{c}$ & $57.5^{c}$ & $44.4^{c}$ & $65.8^{c}$ & $72.1^{b c}$ & $62.8^{c}$ & $66.5^{b}$ & $76.7^{c}$ & $44.0^{b}$ & $64.1^{\mathrm{ab}}$ & $69.3^{d}$ & $83.1^{\mathrm{ab}}$ \\
\hline Peanut meal & $79.2^{\mathrm{a}}$ & $92.9^{\mathrm{a}}$ & $84.4^{a}$ & $87.7^{\mathrm{a}}$ & $89.4^{a}$ & $75.1^{b}$ & $92.6^{a}$ & $92.1^{\mathrm{a}}$ & $76.9^{\mathrm{a}}$ & $81.7^{\mathrm{a}}$ & $89.0^{a}$ & $79.6^{a}$ & $83.4^{\mathrm{a}}$ & $76.9^{\mathrm{a}}$ & $88.8^{\mathrm{a}}$ & $61.1^{\mathrm{a}}$ & $62.3^{\mathrm{ab}}$ & $81.3^{a}$ & $87.4^{a}$ \\
\hline Corn SBM & $79.1^{\mathrm{a}}$ & $89.4^{b}$ & $84.2^{\mathrm{a}}$ & $82.8^{b}$ & $85.5^{b}$ & $83.3^{\mathrm{a}}$ & $90.4^{\mathrm{ab}}$ & $85.7^{b}$ & $75.8^{\mathrm{a}}$ & $81.2^{\mathrm{a}}$ & $80.0^{b}$ & $79.8^{\mathrm{a}}$ & $80.7^{\mathrm{a}}$ & $75.4^{\mathrm{a}}$ & $84.9^{b}$ & $68.6^{a}$ & $77.3^{\mathrm{a}}$ & $80.5^{\mathrm{ab}}$ & $82.1^{a b}$ \\
\hline SEM & 1.0 & 0.7 & 1.4 & 1.1 & 0.9 & 1.2 & 1.0 & 0.9 & 1.2 & 1.5 & 1.1 & 1.4 & 1.1 & 1.3 & 0.9 & 2.0 & 4.6 & 1.3 & 1.4 \\
\hline \multicolumn{20}{|l|}{ Keratinase $(K E)^{2)}$} \\
\hline- & 69.1 & 86.5 & 77.0 & 75.0 & 79.6 & 67.6 & 79.8 & 79.9 & 65.9 & 69.4 & 74.0 & 71.2 & 72.2 & 68.6 & 81.2 & 56.1 & 63.9 & 71.9 & 76.1 \\
\hline+ & 70.1 & 86.6 & 75.4 & 76.1 & 80.5 & 66.4 & 83.5 & 81.3 & 68.0 & 69.0 & 75.5 & 73.2 & 74.0 & 69.9 & 82.6 & 57.1 & 60.7 & 73.3 & 77.8 \\
\hline SEM & 0.6 & 0.4 & 0.8 & 0.6 & 0.5 & 0.7 & 0.6 & 0.5 & 0.7 & 0.9 & 0.6 & 0.8 & 0.7 & 0.8 & 0.5 & 1.2 & 3.2 & 0.7 & 0.8 \\
\hline \multicolumn{20}{|l|}{ p-Value } \\
\hline Diets & $<0.01$ & $<0.01$ & $<0.01$ & $<0.01$ & $<0.01$ & $<0.01$ & $<0.01$ & $<0.01$ & $<0.01$ & $<0.01$ & $<0.01$ & $<0.01$ & $<0.01$ & $<0.01$ & $<0.01$ & $<0.01$ & $<0.01$ & $<0.01$ & $<0.01$ \\
\hline $\mathrm{KE}$ & 0.22 & 0.90 & 0.16 & 0.19 & 0.23 & 0.24 & $<0.01$ & 0.055 & 0.039 & 0.72 & 0.10 & 0.085 & 0.056 & 0.23 & 0.058 & 0.55 & 0.49 & 0.17 & 0.14 \\
\hline Diets $\times K E$ & $<0.01$ & 0.057 & $<0.01$ & $<0.01$ & 0.023 & $<0.01$ & $<0.01$ & 0.026 & 0.019 & $<0.01$ & $<0.01$ & 0.059 & 0.013 & $<0.01$ & 0.032 & 0.25 & 0.27 & 0.11 & $<0.01$ \\
\hline
\end{tabular}

DDGS, distillers dried grains with solubles; SBM, soybean meal; SEM, standard error of means.

1) Data of digestibility were mean of six replicates. Data within a column without common superscripts differ $(p<0.05)$.

2) "." represents diets without keratinase addition, and "+" represents diets with keratinase addition.

Table 6. Standardized ileal digestibility (SID) of crude protein (CP) and amino acids (AA) in 6 experimental diets with or without keratinase addition fed to pigs (\%) ${ }^{1)}$

\begin{tabular}{|c|c|c|c|c|c|c|c|c|c|c|c|c|c|c|c|c|c|c|c|}
\hline Items & $\mathrm{CP}$ & Arg & His & Ile & Leu & Lys & Met & Phe & Thr & Trp & Val & Ala & Asp & Cys & Glu & Gly & Pro & Ser & Tyr \\
\hline \multicolumn{20}{|l|}{ Diets } \\
\hline Rice bran & $54.7^{\mathrm{d}}$ & $82.7^{c}$ & $68.6^{c}$ & $59.2^{\mathrm{e}}$ & $66.6^{e}$ & $64.1^{c}$ & $63.7^{d}$ & $63.0^{d}$ & $55.0^{c}$ & $63.7^{b}$ & $57.0^{d}$ & $60.3^{d}$ & $59.7^{c}$ & $48.5^{c}$ & $70.3^{d}$ & $38.1^{b}$ & $52.2^{b}$ & $57.5^{\mathrm{e}}$ & $60.6^{d}$ \\
\hline Cottonseed meal & $74.3^{b}$ & $91.5^{\mathrm{ab}}$ & $72.4^{\mathrm{bc}}$ & $77.2^{c}$ & $78.4^{d}$ & $63.2^{c}$ & $70.4^{c}$ & $85.7^{b}$ & $68.8^{b}$ & $77.1^{\mathrm{a}}$ & $79.1^{b}$ & $67.5^{c}$ & $80.9^{\mathrm{a}}$ & $73.3^{\mathrm{a}}$ & $86.9^{\mathrm{ab}}$ & $64.2^{\mathrm{a}}$ & $54.1^{b}$ & $76.0^{b c}$ & $79.3^{b}$ \\
\hline Rapeseed meal & $70.6^{b}$ & $83.2^{c}$ & $77.8^{\mathrm{b}}$ & $78.6^{\mathrm{bc}}$ & $79.8^{\text {cd }}$ & $76.0^{b}$ & $87.4^{b}$ & $79.2^{c}$ & $69.8^{b}$ & $68.5^{b}$ & $79.0^{b}$ & $75.7^{\mathrm{ab}}$ & $72.0^{b}$ & $78.1^{\mathrm{a}}$ & $84.9^{b}$ & $66.6^{\mathrm{a}}$ & $68.0^{\mathrm{ab}}$ & $72.2^{\mathrm{cd}}$ & $70.6^{c}$ \\
\hline Corn DDGS & $61.8^{c}$ & $80.7^{c}$ & $70.6^{c}$ & $69.6^{d}$ & $82.0^{c}$ & $42.3^{d}$ & $87.4^{b}$ & $80.1^{c}$ & $58.1^{c}$ & $45.1^{c}$ & $66.2^{c}$ & $72.4^{b c}$ & $63.3^{c}$ & $67.3^{b}$ & $76.9^{c}$ & $44.7^{b}$ & $64.6^{\mathrm{ab}}$ & $69.7^{d}$ & $83.5^{\mathrm{ab}}$ \\
\hline Peanut meal & $79.6^{\mathrm{a}}$ & $93.1^{\mathrm{a}}$ & $84.6^{\mathrm{a}}$ & $88.0^{\mathrm{a}}$ & $89.8^{a}$ & $75.6^{b}$ & $93.5^{\mathrm{a}}$ & $92.5^{\mathrm{a}}$ & $77.5^{\mathrm{a}}$ & $82.1^{\mathrm{a}}$ & $89.5^{\mathrm{a}}$ & $80.2^{\mathrm{a}}$ & $83.6^{\mathrm{a}}$ & $78.1^{\mathrm{a}}$ & $88.9^{\mathrm{a}}$ & $61.7^{\mathrm{a}}$ & $62.4^{\mathrm{ab}}$ & $81.7^{a}$ & $87.9^{\mathrm{a}}$ \\
\hline Corn SBM & $79.5^{\mathrm{a}}$ & $89.6^{b}$ & $84.3^{\mathrm{a}}$ & $83.1^{b}$ & $85.8^{b}$ & $83.6^{a}$ & $90.9^{\mathrm{ab}}$ & $86.1^{b}$ & $76.2^{\mathrm{a}}$ & $81.6^{\mathrm{a}}$ & $80.3^{b}$ & $80.2^{\mathrm{a}}$ & $81.0^{\mathrm{a}}$ & $76.3^{\mathrm{a}}$ & $85.1^{b}$ & $69.5^{\mathrm{a}}$ & $78.0^{\mathrm{a}}$ & $80.8^{a b}$ & $82.6^{\mathrm{ab}}$ \\
\hline SEM & 1.0 & 0.7 & 1.4 & 1.1 & 0.9 & 1.2 & 1.0 & 0.9 & 1.2 & 1.5 & 1.1 & 1.4 & 1.1 & 1.3 & 0.9 & 2.0 & 4.6 & 1.3 & 1.4 \\
\hline \multicolumn{20}{|l|}{ Keratinase $(\mathrm{KE})^{2)}$} \\
\hline - & 69.6 & 86.8 & 77.2 & 75.4 & 80.0 & 68.0 & 80.4 & 80.4 & 66.5 & 69.9 & 74.4 & 71.1 & IL.J & 09.1 & 81.5 & 31.0 & 04.1 & /L.3 & 76.5 \\
\hline+ & 70.6 & 86.8 & 75.5 & 76.5 & 80.8 & 66.9 & 84.1 & 81.8 & 68.6 & 69.5 & 75.9 & 73.7 & 74.3 & 70.9 & 82.9 & 58.0 & 61.6 & 73.7 & 78.3 \\
\hline SEM & 0.6 & 0.4 & 0.8 & 0.6 & 0.5 & 0.7 & 0.6 & 0.5 & 0.7 & 0.9 & 0.6 & 0.8 & 0.7 & 0.8 & 0.5 & 1.2 & 3.2 & 0.7 & 0.8 \\
\hline \multicolumn{20}{|l|}{$p$-value } \\
\hline Diets & $<0.01$ & $<0.01$ & $<0.01$ & $<0.01$ & $<0.01$ & $<0.01$ & $<0.01$ & $<0.01$ & $<0.01$ & $<0.01$ & $<0.01$ & $<0.01$ & $<0.01$ & $<0.01$ & $<0.01$ & $<0.01$ & $<0.01$ & $<0.01$ & $<0.01$ \\
\hline $\mathrm{KE}$ & 0.22 & 0.89 & 0.17 & 0.19 & 0.22 & 0.25 & $<0.01$ & 0.054 & 0.040 & 0.72 & 0.10 & 0.086 & 0.055 & 0.24 & 0.058 & 0.54 & 0.50 & 0.17 & 0.14 \\
\hline Diets $\times K E$ & $<0.01$ & 0.055 & $<0.01$ & $<0.01$ & 0.023 & $<0.01$ & $<0.01$ & 0.026 & 0.020 & $<0.01$ & $<0.01$ & 0.059 & 0.013 & $<0.01$ & 0.032 & 0.24 & 0.26 & 0.11 & $<0.01$ \\
\hline
\end{tabular}

DDGS, distillers dried grains with solubles; SBM, soybean meal; SEM, standard error of means.

1) Data of digestibility were mean of six replicates. Data within a column without common superscripts differ $(p<0.05)$.

2) "." represents diets without keratinase addition, and "+" represents diets with keratinase addition. 
$\mathrm{CP}$ and almost all the AAs except for Arg, Ala, Gly, Pro, and Ser. Among the six tested diets, the cSBM diet and PM diet showed the greatest AID and SID for almost all the AAs, while rice bran diet and corn DDGS diet showed the lowest AID and SID for almost all the AAs.
Effects of KE on the AID of AA in feedstuffs analyzed within each diet in our study are shown in Table 7. Inclusion of KE improved $(\mathrm{p}<0.05)$ the AID of Met, Thr, Val, Asp, Cys, and Tyr in rice bran. The CSM supplemented with KE had greater AID of Met and Val compared with that in CSM without KE

Table 7. Apparent ileal digestibility (AID) of crude protein (CP) and amino acids (AA) in 6 experimental diets with or without keratinase addition fed to pigs analyzed within each diet $(\%)^{1)}$

\begin{tabular}{|c|c|c|c|c|c|c|c|c|c|c|c|c|}
\hline \multirow{2}{*}{ Items } & -2) & $++^{2)}$ & SEM & $p$-value & - & + & SEM & $p$-value & - & + & SEM & $\mathrm{p}$-value \\
\hline & \multicolumn{4}{|c|}{ Rice bran diet } & \multicolumn{4}{|c|}{ Cottonseed meal diet } & \multicolumn{4}{|c|}{ Rapeseed meal diet } \\
\hline$\overline{C P}$ & 51.7 & 56.0 & 1.5 & 0.066 & 71.8 & 75.9 & 1.4 & 0.059 & 68.8 & 71.4 & 1.3 & 0.18 \\
\hline \multicolumn{13}{|c|}{ Indispensable AA } \\
\hline Arg & 82.0 & 82.7 & 0.7 & 0.47 & 90.8 & 92.0 & 0.8 & 0.30 & 81.8 & 84.1 & 1.2 & 0.20 \\
\hline His & 70.5 & 66.2 & 2.4 & 0.23 & 70.8 & 73.7 & 3.3 & 0.55 & 79.6 & 75.6 & 1.0 & 0.022 \\
\hline Leu & 64.6 & 67.5 & 1.0 & 0.097 & 76.2 & 79.9 & 2.0 & 0.22 & 79.9 & 79.1 & 1.6 & 0.75 \\
\hline Ile & 57.9 & 60.9 & 1.3 & 0.13 & 74.8 & 78.9 & 2.2 & 0.20 & 79.4 & 77.2 & 1.8 & 0.41 \\
\hline Lys & 62.0 & 65.2 & 1.6 & 0.18 & 60.5 & 65.0 & 2.4 & 0.21 & 75.5 & 75.9 & 1.2 & 0.81 \\
\hline Met & 54.1 & 71.7 & 2.1 & $<0.01$ & 68.5 & 71.1 & 0.8 & 0.040 & 89.5 & 84.6 & 1.7 & 0.062 \\
\hline Phe & 59.8 & 64.3 & 1.6 & 0.073 & 84.0 & 86.6 & 1.5 & 0.24 & 77.6 & 79.4 & 1.7 & 0.48 \\
\hline Thr & 51.7 & 56.6 & 1.5 & 0.035 & 65.4 & 71.0 & 2.7 & 0.17 & 68.8 & 69.8 & 2.2 & 0.74 \\
\hline Trp & 61.6 & 64.4 & 1.7 & 0.27 & 74.7 & 78.8 & 1.3 & 0.056 & 70.88 & 65.6 & 3.2 & 0.28 \\
\hline Val & 54.6 & 59.2 & 1.0 & $<0.01$ & 74.6 & 82.6 & 1.9 & $<0.05$ & 80.7 & 76.6 & 2.0 & 0.18 \\
\hline \multicolumn{13}{|c|}{ Dispensable AA } \\
\hline Ala & 59.1 & 61.2 & 0.9 & 0.11 & 63.3 & 70.5 & 2.9 & 0.10 & 73.8 & 73.5 & 1.3 & 0.85 \\
\hline Asp & 56.9 & 61.6 & 1.1 & 0.012 & 78.9 & 82.4 & 1.9 & 0.20 & 69.8 & 73.4 & 2.8 & 0.38 \\
\hline Cys & 41.9 & 52.3 & 2.9 & 0.027 & 71.7 & 72.9 & 2.1 & 0.67 & 77.2 & 77.7 & 1.0 & 0.74 \\
\hline Glu & 70.0 & 70.9 & 0.8 & 0.43 & 84.9 & 88.4 & 1.2 & 0.068 & 83.8 & 85.5 & 1.8 & 0.52 \\
\hline Gly & 35.1 & 38.6 & 3.5 & 0.49 & 62.7 & 63.9 & 2.9 & 0.77 & 62.6 & 68.9 & 2.5 & 0.10 \\
\hline Pro & 49.8 & 51.3 & 3.6 & 0.83 & 53.1 & 53.2 & 7.0 & 0.99 & 65.1 & 70.0 & 7.4 & 0.68 \\
\hline Ser & 55.1 & 58.7 & 1.4 & 0.099 & 73.1 & 78.0 & 2.2 & 0.15 & 70.9 & 72.7 & 2.3 & 0.60 \\
\hline \multirow[t]{2}{*}{ Tyr } & 54.6 & 65.1 & 3.3 & 0.042 & 79.6 & 78.2 & 2.2 & 0.67 & 70.4 & 69.7 & 1.9 & 0.81 \\
\hline & \multicolumn{4}{|c|}{ Corn DDGS diet } & \multicolumn{4}{|c|}{ Peanut meal diet } & \multicolumn{4}{|c|}{ Corn SBM diet } \\
\hline$C P$ & 64.2 & 58.3 & 2.0 & 0.057 & 78.1 & 80.3 & 0.7 & 0.054 & 77.5 & 80.7 & 1.0 & 0.048 \\
\hline \multicolumn{13}{|c|}{ Indispensable AA } \\
\hline $\operatorname{Arg}$ & 82.5 & 80.6 & 0.9 & 0.17 & 92.9 & 93.0 & 0.4 & 0.88 & 89.3 & 89.5 & 0.6 & 0.79 \\
\hline His & 75.2 & 65.7 & 1.4 & $<0.01$ & 82.5 & 86.4 & 1.8 & 0.15 & 83.7 & 84.7 & 0.6 & 0.26 \\
\hline Leu & 83.9 & 79.8 & 0.9 & $<0.01$ & 88.5 & 90.4 & 0.6 & 0.046 & 84.7 & 86.4 & 0.4 & 0.022 \\
\hline Ile & 71.8 & 66.7 & 1.2 & 0.013 & 86.3 & 89.0 & 0.7 & 0.015 & 81.4 & 84.2 & 0.6 & 0.010 \\
\hline Lys & 50.6 & 32.5 & 1.9 & $<0.01$ & 74.7 & 75.5 & 1.4 & 0.72 & 82.2 & 84.4 & 1.1 & 0.19 \\
\hline Met & 88.0 & 86.0 & 1.0 & 0.19 & 88.4 & 96.9 & 1.4 & $<0.01$ & 89.9 & 90.9 & 0.9 & 0.45 \\
\hline Phe & 81.5 & 77.7 & 0.9 & 0.011 & 91.4 & 92.9 & 0.5 & 0.066 & 84.8 & 86.6 & 0.5 & 0.021 \\
\hline Thr & 60.3 & 54.7 & 1.5 & 0.022 & 75.2 & 78.5 & 1.1 & 0.057 & 74.0 & 77.6 & 1.1 & 0.040 \\
\hline $\operatorname{Trp}$ & 49.2 & 39.5 & 3.3 & 0.057 & 80.2 & 83.1 & 0.9 & 0.054 & 79.9 & 82.5 & 1.0 & 0.10 \\
\hline Val & 68.0 & 63.5 & 2.0 & 0.14 & 88.5 & 89.6 & 0.7 & 0.24 & 78.7 & 81.2 & 0.9 & 0.073 \\
\hline \multicolumn{13}{|c|}{ Dispensable AA } \\
\hline Ala & 74.7 & 69.4 & 1.3 & 0.013 & 76.2 & 80.3 & 0.8 & $<0.01$ & 78.4 & 81.1 & 1.1 & 0.12 \\
\hline Asp & 65.8 & 59.8 & 1.2 & $<0.01$ & 82.5 & 84.2 & 1.4 & 0.39 & 79.1 & 82.4 & 0.9 & 0.033 \\
\hline Cys & 72.1 & 60.8 & 1.7 & $<0.01$ & 75.2 & 78.6 & 0.9 & 0.021 & 73.7 & 77.2 & 1.3 & 0.075 \\
\hline Glu & 78.8 & 74.6 & 0.8 & $<0.01$ & 87.7 & 89.8 & 1.3 & 0.28 & 83.4 & 86.5 & 1.2 & 0.10 \\
\hline Gly & 47.6 & 39.8 & 3.4 & 0.13 & 60.8 & 61.4 & 1.9 & 0.83 & 67.6 & 69.7 & 2.4 & 0.54 \\
\hline Pro & 65.0 & 63.1 & 5.1 & 0.80 & 73.2 & 49.6 & 7.8 & 0.058 & 77.1 & 77.6 & 3.5 & 0.92 \\
\hline Ser & 71.3 & 67.3 & 1.1 & 0.020 & 79.0 & 80.9 & 0.6 & 0.034 & 78.9 & 82.0 & 0.8 & 0.017 \\
\hline Tyr & 85.3 & 80.9 & 1.0 & 0.011 & 85.0 & 90.3 & 0.8 & $<0.01$ & 81.5 & 82.7 & 1.5 & 0.59 \\
\hline
\end{tabular}

SEM, standard error of means; DDGS, distillers dried grains with solubles; SBM, soybean meal.

1) Data of digestibility were mean of six replicates.

2) "-" represents diets without keratinase addition, and "+" represents diets with keratinase addition. 
addition ( $\mathrm{p}<0.05)$. The KE supplementation decreased $(\mathrm{p}<0.05)$ AID of His in RSM. The AID of all measured AA except for CP, Arg, Met, Trp, Val, Gly, and Pro in KE supplemented corn DDGS were lower $(\mathrm{p}<0.05)$ than that in corn DDGS without KE. Compared with PNM, the KE supplemented PNM had greater $(\mathrm{p}<0.05)$ AID of Leu, Ile, Met, Ala, Cys, Ser, and Tyr. The KE improved $(\mathrm{p}<0.05)$ the AID of CP, Leu, Ile, Phe, Thr, Asp, and Ser in cSBM.

Effects of KE on the SID of AA in feedstuffs analyzed within each diet in our study are shown in Table 8 . Rice bran supple-

Table 8. Standardized ileal digestibility (SID) of crude protein (CP) and amino acids (AA) in 6 experimental diets with or without keratinase addition fed to pigs analyzed within each diet $(\%)^{1)}$

\begin{tabular}{|c|c|c|c|c|c|c|c|c|c|c|c|c|}
\hline \multirow{2}{*}{ Items } & .2) & $++^{2)}$ & SEM & $p$-value & - & + & SEM & $p$-value & - & + & SEM & $p$-value \\
\hline & \multicolumn{4}{|c|}{ Rice bran diet } & \multicolumn{4}{|c|}{ Cottonseed meal diet } & \multicolumn{4}{|c|}{ Rapeseed meal diet } \\
\hline $\mathrm{CP}$ & 52.5 & 56.8 & 1.5 & 0.066 & 72.2 & 76.4 & 1.4 & 0.056 & 69.2 & 71.9 & 1.3 & 0.18 \\
\hline Arg & 82.3 & 83.1 & 0.7 & 0.46 & 90.9 & 92.1 & 0.8 & 0.30 & 82.1 & 84.4 & 1.2 & 0.20 \\
\hline His & 70.7 & 66.4 & 2.4 & 0.23 & 70.9 & 73.8 & 3.3 & 0.55 & 79.7 & 75.8 & 1.0 & 0.023 \\
\hline Leu & 66.0 & 68.0 & 1.0 & 0.19 & 76.6 & 80.3 & 2.0 & 0.22 & 80.2 & 79.5 & 1.6 & 0.76 \\
\hline Met & 55.0 & 72.4 & 2.1 & $<0.01$ & 69.1 & 71.7 & 0.8 & 0.045 & 89.9 & 85.0 & 1.7 & 0.065 \\
\hline Phe & 60.8 & 65.3 & 1.6 & 0.071 & 84.4 & 87.0 & 1.5 & 0.24 & 78.3 & 80.0 & 1.7 & 0.48 \\
\hline Thr & 52.5 & 57.4 & 1.5 & 0.035 & 66.0 & 71.6 & 2.7 & 0.17 & 69.2 & 70.3 & 2.2 & 0.73 \\
\hline Trp & 62.3 & 65.0 & 1.7 & 0.28 & 75.1 & 79.2 & 1.3 & 0.056 & 71.2 & 65.9 & 3.2 & 0.28 \\
\hline Val & 55.3 & 59.9 & 1.0 & $<0.01$ & 75.1 & 83.0 & 1.9 & 0.015 & 81.0 & 77.0 & 2.0 & 0.18 \\
\hline Glu & 70.5 & 71.4 & 0.8 & 0.42 & 85.1 & 88.6 & 1.2 & 0.068 & 84.0 & 85.7 & 1.8 & 0.51 \\
\hline Gly & 36.3 & 39.9 & 3.5 & 0.48 & 63.6 & 64.9 & 2.9 & 0.76 & 63.4 & 69.7 & 2.5 & 0.10 \\
\hline Pro & 51.3 & 53.1 & 3.6 & 0.80 & 54.0 & 54.3 & 7.0 & 0.98 & 65.7 & 70.2 & 7.4 & 0.67 \\
\hline Ser & 55.7 & 59.4 & 1.4 & 0.098 & 73.5 & 78.4 & 2.2 & 0.15 & 71.3 & 73.1 & 2.3 & 0.59 \\
\hline \multirow[t]{2}{*}{ Tyr } & 55.3 & 65.8 & 3.3 & 0.042 & 79.9 & 78.6 & 2.2 & 0.69 & 70.9 & 70.3 & 1.9 & 0.83 \\
\hline & \multicolumn{4}{|c|}{ Corn DDGS diet } & \multicolumn{4}{|c|}{ Peanut meal diet } & \multicolumn{4}{|c|}{ Corn SBM5 diet } \\
\hline$C P$ & 64.7 & 58.8 & 2.0 & 0.057 & 78.5 & 80.8 & 0.7 & 0.053 & 78.0 & 81.1 & 1.0 & 0.048 \\
\hline \multicolumn{13}{|c|}{ Indispensable AA } \\
\hline $\operatorname{Arg}$ & 82.8 & 80.9 & 0.9 & 0.17 & 93.0 & 93.1 & 0.4 & 0.90 & 89.5 & 89.8 & 0.6 & 0.78 \\
\hline Trp & 50.0 & 40.3 & 3.3 & 0.057 & 80.7 & 83.6 & 0.9 & 0.058 & 80.2 & 82.9 & 1.0 & 0.098 \\
\hline Val & 68.5 & 64.0 & 2.0 & 0.14 & 88.9 & 90.0 & 0.7 & 0.27 & 79.0 & 81.6 & 0.9 & 0.074 \\
\hline \multicolumn{13}{|c|}{ Dispensable AA } \\
\hline Ala & 75.0 & 69.7 & 1.3 & 0.013 & 76.7 & 80.8 & 0.8 & $<0.01$ & 78.8 & 81.5 & 1.1 & 0.12 \\
\hline Asp & 66.3 & 60.3 & 1.2 & $<0.01$ & 82.7 & 84.5 & 1.4 & 0.40 & 79.3 & 82.6 & 0.9 & 0.034 \\
\hline Cys & 73.0 & 61.7 & 1.7 & $<0.01$ & 76.4 & 79.8 & 0.9 & 0.021 & 74.6 & 78.1 & 1.3 & 0.076 \\
\hline Glu & 79.0 & 74.9 & 0.8 & $<0.01$ & 87.9 & 90.0 & 1.3 & 0.28 & 83.6 & 86.7 & 1.2 & 0.097 \\
\hline Gly & 48.7 & 40.8 & 3.4 & 0.13 & 61.4 & 62.0 & 1.9 & 0.84 & 68.5 & 70.6 & 2.4 & 0.54 \\
\hline Pro & 65.5 & 63.6 & 5.1 & 0.80 & 74.0 & 50.4 & 7.8 & 0.057 & 77.8 & 78.2 & 3.5 & 0.93 \\
\hline Ser & 71.7 & 67.6 & 1.1 & 0.020 & 79.3 & 81.2 & 0.6 & 0.037 & 79.2 & 82.3 & 0.8 & 0.017 \\
\hline Tyr & 85.7 & 81.3 & 1.0 & 0.012 & 85.4 & 90.5 & 0.8 & $<0.01$ & 82.0 & 83.2 & 1.5 & 0.59 \\
\hline
\end{tabular}

SEM, standard error of means; DDGS, distillers dried grains with solubles; SBM, soybean meal.

1) Data of digestibility were mean of six replicates.

2) "_" represents diets without keratinase addition, and "+" represents diets with keratinase addition. 
mented with KE had greater SID of Met, Thr, Val, Asp, Cys, and Tyr for pigs $(\mathrm{p}<0.05)$. The KE inclusion had greater $(\mathrm{p}<$ 0.05) SID of Met and Val in CSM. Similar to the effect on AID of AA, KE decreased $(\mathrm{p}<0.05)$ the SID of His in RSM. The SID of all measured AA except for Arg, Met, Trp, Val, Gly, and Pro were lower $(\mathrm{p}<0.05)$ in corn DDGS with KE supplementation than without. The SID of Leu, Ile, Met, Ala, Cys, Ser, and Tyr in PNM including KE were greater $(\mathrm{p}<0.05)$ than that in PNM without KE. The KE inclusion to the CSBM diet improved $(p<0.05)$ the SID of CP, Leu, Ile, Phe, Thr, Asp, and Ser.

\section{DISCUSSION}

In our study, the chemical composition of the rice bran was within the ranges reported by Shi et al [22]. The chemical composition of the CSM was within the ranges reported by Li et al [23]. The chemical composition of the RSM was within the ranges reported by Li et al [24]. The chemical composition of the corn DDGS was comparable to those values from NRC [25] (corn DDGS containing greater than $6 \%$ oil but less than 9\% oil) and were within the ranges reported by Li et al [26]. The chemical composition of the PNM was comparable with the values reported by Li et al [27] and NRC [25]. The concentration of $\mathrm{CP}(47.46 \%$ as fed) in SBM was little higher than that of $45.1 \%$ reported by Lagos and Stein [28]. The concentration of individual AA in each tested feedstuff was within the relative standard deviation presented in NRC [25]. The values of endogenous $\mathrm{CP}$ and AA losses presented in Table 3 were comparable with the values from previous studies that also conducted in our lab $[20,24,27]$.

The KEs are mainly serine and metalloproteases, which could hydrolyze protein without being denatured or broken down by the gastrointestinal tract in broiler chickens [9]. It may also need the presence of live cells for activating the sulfitolysis or reduction in disulfide bonds and proteolysis effect of KE $[29,30]$. Though the proteolysis effection of most proteases may begin when the animals consumed the feed, most digestive enzymes are not stable at the low $\mathrm{pH}$ encountered in the stomach and upper small intestine. As a result, these enzymes may become inactivated, or a portion of the enzyme is inactivated. This process of inactivation is the likely cause of the variable results of other enzymes reported previously. Microbial KEs are predominantly extracellular when grow on keratinous substrates and can be stable over a wide range of $\mathrm{pH}$ from 5 to 13 [31]. The optimal $\mathrm{pH}$ for KE produced by Bacillus licheniformis PWD-1 was about 7.5 [2], which is like the conditions in the lower jejunum and ileum of pigs [32]. Therefore, the KE included in the current study was likely exerting its effect on protein digestion in the lower small intestine.

The significant interaction effect between feedstuff and $\mathrm{KE}$ supplementation indicated that the effect of KE on AA digestibility can be influenced by treatment diets. Considering the more abundant AA contents in cSBM diet and PM diet, and the relative poor AA contents in rice bran diet and corn DDGS diet, it is reasonable to observe the greatest AID and SID of almost all the AAs in cSBM and PM diets, while the lowest AID and SID of almost all the AAs in rice bran and corn DDGS diets. The improved AID of CP and AA in the KE supplemented cSBM diet agreed with Wang et al [7] where the same level of KE (0.05\%) supplementation in a cSBM basal diet significantly increased the dietary AID of CP, Arg, His, Leu, Phe, and Thr. About $40 \%$ and $30 \%$ of the total soybean globulin proteins were glycinin and $\beta$-conglycinin, respectively $[11,12]$. The ratio of CP provided by SBM vs corn was 2.1:1 in CSBM diet used in our trial. Therefore, the improved digestibility of $\mathrm{CP}$ and $\mathrm{AA}$ in the KE added cSBM-based diet is most likely due to the digestion of glycinin and $\beta$-conglycinin, which were done via protein-disulfide reductase break down of the cystine disulfide bond and the peptidohydrolase hydrolyzation of the denatured protein into peptides and AA [31,33]. The KE has been used to improve digestibility of feather meal in livestock diets [34] and in poultry diets $[8,10,35]$. However, no previous studies reported the utilization of KE on the other feed ingredients.

The KE significantly improved the AID and SID of Met in rice bran, CSM, and PNM but not in RSM and CSBM. We supposed that this is related to the relative lower concentration of Met in the former three feedstuffs, considering relative less disulfide bonds that KE may need to break down. In rice bran, both the AID and SID of Met and Cys improved more than $20 \%$, which can also primarily attribute to break down effect of KE on disulfide bonds of Met and Cys. In CSM, KE only affected the AID and SID of Met and Val, but not the dispensable AA. In RSM, KE supplementation only improved the AID and SID of His. In addition to Met, KE also improved the AID and SID of branched AA of Leu and Ile and some dispensable AA in PNM.

Although KE supplementation significantly improved the AID and SID of some AA in the five detected feedstuffs, it only significantly increased the $\mathrm{CP}$ digestibility in $\mathrm{CSBM}$. In the current study, the CP levels in the detected diets except for rice bran diet were similar to those reported by Wang et al [7], who also found that there was no interaction effect between the dietary KE $(0.05 \%$ vs $0.10 \%)$ and CP $(22.0 \%$ vs $20.0 \%)$. Therefore, the various $\mathrm{CP}$ levels may not explain the discrepancies of the KE supplementation effects on $\mathrm{CP}$ digestibility in different feedstuffs. Moreover, the relative lower digestibility of Lys in corn DDGS compared with that in the other feedstuffs was reasonable, because of the heat damage during fermentation or drying during the processing of DDGS [36, 37]. However, the reasons for the reduction of AID and SID of most AA in corn DDGS supplemented with KE were not known. This is a new finding that has never been reported before. The possible hypothesis may be that some components 
in corn DDGS may have negative effects on activity of KE. Future studies are needed to explore the underlying mechanisms.

\section{CONCLUSION}

This study indicated that KE had different effects on the ileal AA digestibility of feedstuffs for growing pigs. In corn DDGS, KE reduced the ileal digestibility for almost all the AAs. These findings could give some directions for KE utilization in swine feed containing CSBM, corn DDGS, CSM, RSM, PNM, and rice bran.

\section{CONFLICT OF INTEREST}

We certify that there is no conflict of interest with any financial organization regarding the material discussed in the manuscript. Zhang B and Sun B are employees of Novus International, Inc. company.

\section{ACKNOWLEDGMENTS}

The KE was provided by Novus International, Inc. Thanks for their support. This research was financially supported by the Modern Agricultural Industry Technology System (CARS36), Developing key equipment for digital management and monitoring environment in animal production (2013AA10230 602), National Natural Science Foundation of China (31372317) and the 111 Project (B16044).

\section{REFERENCES}

1. Williams CM, Richter CS, MacKenzie JMJr, Shih JCH. Isolation, identification and characterization of a feather-degrading bacterium. Appl Environ Microbiol 1990;56:1509-15.

2. Lin X, Lee CG, Casale ES, Shih JCH. Purification and characterization of a keratinase from a feather-degrading Bacillus licheniformis strain. Appl Environ Microbiol 1992;58:3271-5.

3. Shih JCH, Wang JJ. Keratinase technology: from feather degradation and feed additive to prion destruction. CAB Reviews: Perspectives in Agriculture, Veterinary Science, Nutrition and Natural Resources. Wallingford, UK: CABI; 2006. No.042.

4. Yu RJ, Ragot J, Blank F. Keratinases: hydrolysis of keratinous substrates by three enzymes of Trichophyton mentagrophytes. Experientia 1972;28:1512-3.

5. Lin X, Shih JCH, Swaisgood HE. Hydrolysis of feather keratin by immobilized keratinase. Appl Environ Microbiol 1996;62: 4273-5.

6. Gradišar H, Friedrich J, Križaj I, Jerala R. Similarities and specificities of fungal keratinolytic proteases: Comparison of keratinases of Paecilomyces marquandii and Doratomyces microsporus to some known proteases. Appl Microbiol Biotechnol 2005;71:3420-6.
7. Wang D, Zeng Z, Piao XS, et al. Effects of keratinase supplementation of corn-soybean meal based diets on apparent ileal amino acid digestibility in growing pigs and serum amino acids, cytokines, immunoglobulin levels and loin muscle area in nursery pigs. Arch Anim Nutr 2011;65:290-302.

8. Odetallah NH, Wang JJ, Garlich JD, Shih JCH. Keratinase in starter diets improves growth of broiler chicks. Poult Sci 2003; 82:664-70.

9. Wang JJ, Garlich JD, Shih JCH. Beneficial effects of versazyme, a keratinase feed additive, on body weight, feed conversion, and breast yield of broiler chickens. J Appl Poult Res 2006; 15:544-50.

10. Wang H, Guo Y, Shih JCH. Effects of dietary supplementation of keratinase on growth performance, nitrogen retention and intestinal morphology of broiler chickens fed diets with soybean and cottonseed meals. Anim Feed Sci Technol 2008;140:37684.

11. Brandon DL, Friedman M. Immunoassays of soy proteins. J Agric Food Chem 2002;50:6635-42.

12. Sun P, Li DF, Li ZJ, Dong B, Wang FL. Effects of glycinin on IgE-mediated increase of mast cell numbers and histamine release in the small intestine. J Nutr Biochem 2008;19:627-33.

13. Zhao Y, Qin G, Sun Z, et al. Disappearance of immunoreactive glycinin and $\beta$-conglycinin in the digestive tract of piglets. Arch Anim Nutr 2008;62:322-30.

14. Wang JJ, Shih J. Fermentation production of keratinase from Bacillus licheniformis PWD-l and a recombinant B. subtillis FDB-29. J Ind Microbiol Biothnol 1999;22:608-16.

15. Gradišar H, Kern S, Friedrich J. Keratinase of Doratomyces microspores. Appl Microbiol Biotechnol 2000;53:196-200.

16. Stein HH, Shipley CF, Easter RA. Technical note: A technique for inserting a T-cannula into the distal ileum of pregnant sows. J Anim Sci 1998;76:1433-6.

17. AOAC. Official methods of analysis: Association of Official Analytical Chemists, 18th edn. Washington DC, USA; AOAC International; 2005.

18. Thiex NJ, Anderson S, Gildemeister B. Crude fat, diethyl ether extraction, in feed, cereal grain, and forage (Randall/Soxtec/ submersion method): Collaborative study. J AOAC Int 2003; 86:888-98.

19. Van Soest PJ, Robertson JB, Lewis BA. Methods for dietary fiber neutral detergent fiber and nonstarch polysaccharides in relation to animal nutrition. J Dairy Sci 1991;74:3583-97.

20. Li PL, Wu F, Chen Y, et al. Determination of the energy content and amino acid digestibility of double-low rapeseed cakes fed to growing pigs. Anim Feed Sci Technol 2015;210:243-53.

21. Stein HH, Sève B, Fuller MF, Moughan PJ, de Lange CFM. Invited review: Amino acid bioavailability and digestibility in pig feed ingredients: Terminology and application. J Anim Sci 2007;85:172-80.

22. Shi CX, Liu ZY, Shi M, et al. Prediction of digestible and metabolizable energy content of rice bran fed to growing pigs. 
Asian-Australas J Anim Sci 2015;28:654-61.

23. Li JT, Li DF, Zang JJ, et al. Evaluation of energy digestibility and prediction of digestible and metabolizable energy from chemical composition of different cottonseed meal sources fed to growing pigs. Asian-Australas J Anim Sci 2012;25:14308.

24. Li PL, Wang FL, Wu F, et al. Chemical composition, energy and amino acid digestibility in double-low rapeseed meal fed to growing pigs. J Anim Sci Biotechnol 2015;6:37.

25. NRC. Nutrient requirements of swine. 11th ed. Washington, DC, USA: National Academy Press; 2012.

26. Li P, Li DF, Zhang HY, et al. Determination and prediction of energy values in corn distillers dried grains with solubles sources with varying oil content for growing pigs. J Anim Sci 2015;93:3458-70.

27. Li QY, Piao XS, Liu JD, et al. Determination and prediction of the energy content and amino acid digestibility of peanut meals fed to growing pigs. Arch Anim Nutr 2014;68:196-210.

28. Lagos LV, Stein HH. Chemical composition and amino acid digestibility of soybean meal produced in the United States, China, Argentina, Brazil, or India. J Anim Sci 2017;95:162636.

29. Böckle B, Muller R. Reduction of disulfide bonds by Streptomyces pactum during growth on chicken feathers. Appl Environ Microbiol 1997;63:790-2.

30. Ramnani P, Singh R, Gupta R. Keratinolytic potential of Bacillus licheniformis RG1: structural and biochemical mechanism of feather degradation. Can J Microbiol 2005;51:191-6.

31. Gupta R, Ramnani P. Microbial keratinases and their prospective applications: an overview. Appl Microbiol Biotechnol 2006; 70:21-33.

32. Braude R, Fulford RJ, Low AG. Studies on digestion and absorption in the intestines of growing pigs. Measurements of the flow of digesta and pH. Br J Nutr 1976;36:497-510.

33. Tu GQ, Ye YJ, Zhang B, Guo MJ, Sun TH. Studies on the biomechanism of keratin decomposed by streptomyces. Acta Agric Univ Jiangxiensis 1998;20:1-5.

34. Kim WK, Patterson PH. Nutritional value of enzyme or sodium hydroxide treated feathers from dead hens. Poult Sci 2000;79: 528-34.

35. Lee GG, Ferket PR, Shih JCH. Improvement of feather digestibility by bacterial keratinase as a feed additive. FASEB J 1991; 59:1312.

36. Pahm AA, Pedersen C, Stein HH. Application of the reactive lysine procedure to estimate lysine digestibility in distillers dried grains with solubles fed to growing pigs. J Agric Food Chem 2008;56:9441-6.

37. Kim BG, Kil DY, Zhang Y, Stein HH. Concentrations of analyzed or reactive lysine, but not crude protein, may predict the concentration of digestible lysine in distillers dried grains with solubles fed to pigs. J Anim Sci 2012;90:3798-808. 